
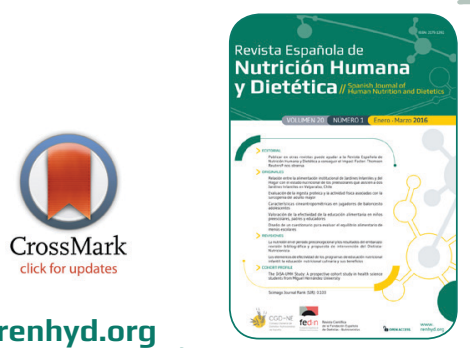

www.renhyd.org

ORIGINAL

\title{
Valoración de la efectividad de la educación alimentaria en niños preescolares, padres y educadores
}

\author{
Susana Rodrigo-Cano ${ }^{a}$, José Miguel Soriano ${ }^{a}$, Joaquín Aldas-Manzano ${ }^{b, *}$ \\ a Departamento de Medicina Preventiva y Salud Pública, Facultat de Farmàcia, Universitat de València, España. \\ b Departamento de Comercialización e Investigación de Mercados, Facultat d’Economia, Universitat de València, España. \\ *joaquin.aldas@uv.es
}

Recibido el 13 de septiembre de 2015; aceptado el 15 de enero de 2016.

\section{PALABRAS CLAVE}

Educación;

Niño preescolar;

Dieta;

Hábitos alimentarios;

Dieta Mediterránea;

Educadores de la salud.
Valoración de la efectividad de la educación alimentaria en niños preescolares, padres y educadores

\section{RESUMEN}

Introducción: La etapa infantil es el momento en el que se desarrollan los factores de riesgo que pueden desencadenar en edades adultas enfermedades relacionadas con la alimentación. Éste es el momento adecuado para instaurar las bases de una alimentación saludable. El objetivo del presente estudio fue evaluar la adherencia a la Dieta Mediterránea de niños, los conocimientos sobre hábitos saludables de niños, padres y profesores antes y después de una intervención de educación alimentaria, así como evaluar su eficacia.

Material y Métodos: Estudio transversal en el que participaron 94 estudiantes de edades comprendidas entre 3 y 6 años, 12 padres y 8 profesores. Se calculó el grado de conocimientos sobre hábitos saludables mediante cuestionarios ad hoc de todos ellos y la adherencia a la Dieta Mediterránea de los niños mediante el Test de Calidad de la Dieta Mediterránea en la infancia y la adolescencia.

Resultados: Se consiguió un aumento significativo en los conocimientos globales sobre hábitos saludables ( $t=-6,29 ; p<0,001)$, así como los referentes al número de comidas $(t=-2,35 ; p<0,05)$ y frecuencia de consumo de fruta $(t=-3,92 ; p<0,01)$, verdura $(t=-2,35 ; p<0,05)$ y pescado $(t=-7,42 ; p<0,001)$ de los padres y en los conocimientos sobre actividad física $(t=-2,58 ; p<0,05)$ de los niños. Los padres de los niños con mayor adherencia a la Dieta Mediterránea mejoraron sus conocimientos sobre hábitos saludables de manera significativa $(\rho=0,75 ; p<0,01)$.

Conclusiones: Son necesarias intervenciones educativas dirigidas a niños, padres y educadores para aumentar los conocimientos sobre alimentación saludable. Con intervenciones de educación alimentaria se consigue una mejora en los conocimientos de los padres, principales responsables de la alimentación de los menores. 
$\sum$ Effectiveness assessment of food education among preschool children, parents and educators

\section{KEYWORDS}

Education;

Child, Preschool;

Diet;

Food Habits;

Diet, Mediterranean;

Health Educators.

\section{ABSTRACT}

Introduction: Childhood is the period in which risk factors that can start food diseases in adults are developed. Therefore this is the appropriate moment to set up the values of a healthy diet. The main aim of this study was to evaluate the adherence to the Mediterranean Diet in children and also the knowledge about healthy habits in children, parents and teachers before and after food education intervention as well as evaluating its effectiveness.

Material and Methods: Cross-sectional study that included 94 students aged between 3 and 6 years old, 12 parents and 8 teachers. The level of healthy habits knowledge was determined with ad hoc questionnaires for all of them whereas the adherence to the Mediterranean Diet was determined with the Quality Test of the Mediterranean Diet on Childhood and Adolescence in children.

Results: It has been reached a significant increase in global healthy habits knowledge ( $t=-6.29$; $\mathrm{p}<0.001)$, in how often they have to eat $(t=-2.35 ; p<0.05)$, as well as in fruit $(t=-3.92 ; p<0.01)$, vegetables $(t=-2.35 ; p<0.05)$ and fish $(t=-7.42 ; p<0.001)$ frequency intake in parents and also in physical activities knowledge $(t=-2.58 ; \mathrm{p}<0.05)$ in children. Moreover, children's parents with more adhesion to the Mediterranean Diet improved their healthy habits knowledge significantly $(\rho=0.75 ; p<0.01)$.

Conclusions: Education interventions for children, parents and educators are necessary in order to increase healthy food knowledge. With food education interventions is possible to get a significant improvement in the parents' knowledge, the main responsible for their children feeding.

\section{CITA}

Rodrigo-Cano S, Soriano JM, Aldas-Manzano J. Valoración de la efectividad de la educación alimentaria en niños preescolares, padres y educadores. Rev Esp Nutr Hum Diet. 2016; 20(1): 32 - 39. D0I: 10.14306/renhyd.20.1.182

\section{INTRODUCCIÓN}

La alimentación incide en el crecimiento, desarrollo y salud de la población. Una alimentación incorrecta se relaciona con una mayor prevalencia de enfermedades crónicas relacionadas con la alimentación $n^{1,2}$, siendo la etapa infantil cuando se desarrollan los factores de riesgo de estas enfermedades que normalmente se pronuncian en edades adultas 3 ; además es la etapa en la que se adquieren los hábitos alimentarios, influidos por padres pero también por profesores ${ }^{4}$. Actualmente la Dieta Mediterránea destaca por ser un estilo de vida con uno de los patrones alimentarios más saludables ${ }^{5,6}$, caracterizado por un consumo elevado de aceite de oliva, fruta, verdura y cereales, un consumo moderado de pescado y carne blanca y un bajo consumo de lácteos, carnes rojas y procesadas y dulces; convirtiéndola en una dieta rica en antioxidantes y pobre en grasas saturadas $^{7}$. Relacionándose una elevada adherencia a la Dieta Mediterránea con una baja mortalidad y un efecto protector frente enfermedades cardiovasculares, diabetes tipo 2 y algún tipo de cáncer ${ }^{6-9}$. Este conjunto de beneficios contrasta con una disminución de su seguimiento, lo que justifica su promoción entre la población ${ }^{10,11}$. Anteriores estudios ponen de manifiesto que pequeñas intervenciones nutricionales realizadas en la escuela, contando con la colaboración de profesores y padres, mejoran los conocimientos sobre alimentación o los hábitos alimentarios ${ }^{12,13}$. El objetivo de este estudio es evaluar la adherencia a la Dieta Mediterránea de niños preescolares y los conocimientos sobre hábitos saludables de estos, sus padres y profesores, que incluyen la alimentación y la actividad física, antes y después de realizar una intervención de educación alimentaria, así como valorar su eficacia. 


\section{• \\ MATERIAL Y MÉTODOS}

El estudio se llevó a cabo en un colegio concertado de la Comunidad Valenciana con niños de edades comprendidas entre 3-6 años durante el curso académico 2014-2015, cumpliendo con los principios éticos definidos en la Declaración de Helsinki y autorizado por el Comité Ético de Investigaciones en Humanos de la Universitat de València.

Se trató de un estudio descriptivo y transversal formado por una muestra de niños de la etapa de Educación Infantil, padres y profesores. Previo a la realización del estudio cada participante entregó el correspondiente consentimiento informado que cumplía con la legislación vigente en España ${ }^{14}$.

El estudio se dividió en dos fases, transcurriendo un mes entre cada una de ellas, tiempo suficiente para la ejecución de una intervención que tuvo como objetivo modificar la alimentación de los niños. Cada fase estuvo compuesta por un taller y una charla.

Los talleres de ambas fases dirigidos a los niños, tuvieron una duración de 40 minutos. En ellos se explicaron las pautas básicas de una alimentación saludable utilizando como guía la Pirámide de los Alimentos ${ }^{15}$ así como la importancia de realizar actividad física. Se realizaron juegos educativos haciendo uso de la manipulación de objetos para conseguir un aprendizaje más profundo ${ }^{16}$. Antes de comenzar el primer taller y tras finalizar el segundo, los niños rellenaron un cuestionario realizado ad hoc para evaluar sus conocimientos sobre hábitos saludables que incluyeron la alimentación y la actividad física. Además en los talleres estuvieron los profesores, quienes rellenaron también un cuestionario realizado ad hoc para evaluar sus conocimientos sobre hábitos saludables antes de comenzar el primer taller y tras finalizar el segundo.

Las dos charlas, de 60 minutos de duración estuvieron dirigidas a los padres de los niños que quisieron participar. En ellas se trabajó la educación alimentaria utilizando también como guía la Pirámide de los Alimentos ${ }^{15}$. También en este caso, antes de iniciar la primera charla y tras finalizar la segunda, los padres cumplimentaron el mismo cuestionario que los profesores para evaluar sus conocimientos sobre hábitos saludables y otro ad hoc para evaluar hábitos alimentarios de sus hijos. Para evaluar la adherencia a la Dieta Mediterránea que tenían, sus hijos respondieron el Test de Calidad de la Dieta Mediterránea en la Infancia y la Adolescencia ${ }^{17}$. Se trata de un cuestionario de 16 preguntas donde las respuestas negativas adquieren valor $0 \mathrm{y}$ las respuestas afirmativas adquieren un valor de +1 ó -1 en función de si suponen una connotación positiva o negativa a la Dieta Mediterránea. La puntuación obtenida da lugar al índice Kidmed que clasifica la dieta en: dieta de muy baja calidad o baja adherencia (índice Kidmed entre 0-3), necesidad de mejorar el patrón alimentario para ajustarlo al modelo mediterráneo o adherencia media (índice Kidmed entre 4-7), Dieta Mediterránea óptima o adherencia alta (índice Kidmed entre 8-12) ${ }^{17}$.

Los cuestionarios para evaluar los conocimientos sobre hábitos saludables de padres y profesores y para evaluar los hábitos alimentarios de los niños fueron evaluados previamente en una muestra ajena al estudio pero de características similares, realizándose los cambios pertinentes en los cuestionarios de manera que éstos se adaptaran lo máximo posible a la muestra de estudio real.

Las variables que miden la adherencia a la Dieta Mediterránea se codificaron como constata el Test de Calidad de la Dieta Mediterránea en la Infancia y la Adolescencia.

Los análisis estadísticos se realizaron con SPSS versión 21. Las variables que miden el conocimiento sobre hábitos saludables y hábitos alimentarios se codificaron como 0 cuando la asunción era errónea y como 1 cuando era acertada. Por lo tanto, los niveles de acierto se muestran como media desviación típica pudiendo interpretarse esta media como porcentaje de acierto. Para evaluar la eficacia de la intervención, las medias de aciertos pre- y posintervención se analizaron mediante un Test t para muestras relacionadas. El nivel de consideración estadística se estableció en $p<0,05$.

\section{RESULTADOS}

De los 235 alumnos de Educación Infantil, la muestra final de estudio para evaluar los conocimientos de hábitos saludables de los niños estuvo compuesta por 94 individuos entre 3 y 6 años: 23,4\% estudiantes de primer curso de Educación Infantil; $39,36 \%$ estudiantes de segundo curso de Educación Infantil; y 37,23\% estudiantes de tercer curso de Educación Infantil.

Los aciertos medios del primer cuestionario que rellenaron los niños, como muestra la Tabla 1, fueron de 1,72. Dado que se trató de cuatro preguntas codificadas como 0 en caso de error y 1 en caso de acierto, el rango de variación teórica de esta variable fue $0-4$. Por tanto, el número total de aciertos estuvo por debajo del $50 \%$. El hábito que más claro tuvieron, acertado por el $65 \%$ de la muestra, fue que debe realizarse otra actividad física diferente a caminar o correr. En cuanto a hábitos alimentarios, en primer lugar, un $49 \%$ de la muestra sabía que se debe tomar fruta tres veces al día; 
Tabla 1. Conocimientos sobre hábitos saludables de los niños.

\begin{tabular}{|c|c|c|c|c|}
\hline \multirow[b]{3}{*}{ Pregunta } & \multicolumn{2}{|c|}{ Aciertos* } & \multirow[b]{3}{*}{$\mathbf{t}$} & \multirow[b]{3}{*}{ Sig.** } \\
\hline & Preintervención & Posintervención & & \\
\hline & media $\pm D T^{* *}$ & media $\pm D T$ & & \\
\hline ¿Qué es un desayuno completo? & $0,35 \pm 0,48$ & $0,33 \pm 0,47$ & 0,36 & $>0,05$ \\
\hline ¿Qué debe incluir la comida diariamente? & $0,23 \pm 0,43$ & $0,29 \pm 0,46$ & $-0,84$ & $>0,05$ \\
\hline ¿Cuántas veces se debe tomar fruta al día? & $0,49 \pm 0,50$ & $0,46 \pm 0,50$ & 0,56 & $>0,05$ \\
\hline ¿Qué actividades se deben realizar durante la semana? & $0,65 \pm 0,48$ & $0,79 \pm 0,41$ & $-2,58$ & $<0,05$ \\
\hline Aciertos totales & $1,72 \pm 0,92$ & $1,86 \pm 1,10$ & $-1,21$ & $>0$ \\
\hline
\end{tabular}

$\mathbf{n}_{\text {niños }}=94 ;$; Codificación: 1= acierto, 0= fallo; **DT: desviación típica; ***Sig.: significación estadística.

en segundo lugar, un 35\% sabía que un desayuno completo está formado por un lácteo, un hidrato de carbono y una fruta; y en tercer lugar, un $23 \%$ de la muestra sabía que la comida debe incluir diariamente verdura, un plato principal y fruta. Tras los talleres de intervención, la media global de aciertos del segundo cuestionario subió a 1,86; no siendo este crecimiento significativo $(t=-1,21 ; p>0,05)$. Si se analizan las preguntas de manera separada, sólo las actividades que deben realizarse durante la semana experimentaron un crecimiento significativo en el número de aciertos, aumentando un $14 \%(t=-2,58 ; p<0,05)$. La intervención resultó más efectiva en las niñas $(1,51)$, dado que al principio el conocimiento de éstas fue significativamente inferior al de los niños $(1,89)(t=1,99 ; p<0,05)$, mientras que al final de la intervención esta diferencia dejó de ser significativa ( $t=1,39$; $p>0,05)$.

El cuestionario para evaluar los conocimientos sobre hábitos saludables de padres y profesores fue el mismo, como refleja la Tabla 2. La muestra para evaluar los conocimientos de los profesores estuvo compuesta por 8 individuos. Los aciertos medios del primer cuestionario que rellenan éstos son de 7,50. Dado que se trató de diez preguntas codificadas como 0 en caso de error y 1 en caso de acierto, el rango de variación teórica de esta variable fue 0-10. Por tanto, el número total de aciertos estuvo por encima del 50\%. El 100\% de los profesores en ese momento conocía cuántas raciones de verdura deben tomarse al día, cuál es el aceite más saludable, cuántas veces debe realizarse actividad física a lo largo de la semana y que no es recomendable tomar golosinas, snacks, refrescos o zumos a diario. Tras las intervenciones, la media global de aciertos del segundo cuestionario ascendió a 7,75 , suponiendo un aumento no significativo $(t=-0,68$; $p>0,05)$. Sin embargo, aumentó en un $12 \%$ el número de profesores que conocía cuántas comidas deben realizarse al día o cuántas veces deben tomarse legumbres ( $t=-1,00$; $p>0,05$ en ambos casos), un $37 \%$ la frecuencia de consumo de pescado $(t=-2,05 ; p>0,05)$ y un $38 \%$ la frecuencia de consumo de fruta $(t=-1,43 ; p>0,05)$ aunque también de manera no significativa $(p>0,05)$.

En el caso de los padres, la muestra para evaluar sus conocimientos estuvo compuesta por 12 individuos. Los aciertos medios del primer cuestionario, como se observa en la Tabla 2, son de 6,67, estando el número total de aciertos por encima del $50 \%$. Todos sabían que el aceite de oliva es el más saludable y que no es recomendable tomar golosinas, snacks, refrescos o zumos comerciales a diario. Tras las intervenciones, la media global de aciertos del segundo cuestionario ascendió a 8,83 , siendo un crecimiento significativo ( $t=-6,29 ; p<0,001$ ). Analizando detalladamente las preguntas, experimentaron un aumento significativo en el número de aciertos las referentes a cuántas comidas deben realizarse al día y la frecuencia de consumo de verdura, que aumentaron un 33\% ambas ( $t=-2,35 ; \mathrm{p}<0,05$ en ambos casos); frecuencia de consumo de fruta, que aumentó un $59 \%$ $(t=-3,92 ; p<0,01) ; y$ frecuencia de consumo de pescado, que aumentó un $83 \%(t=-7,42 ; p<0,001)$.

La muestra final en la que se evaluó la adherencia a la Dieta Mediterránea de niños estuvo compuesta por 12 padres: $50 \%$ padres de estudiantes de primer curso de Educación Infantil, 8,33\% padres de estudiantes de segundo curso de Educación Infantil y $41,66 \%$ padres de estudiantes de tercer curso de Educación Infantil. Previo a la intervención educativa, la muestra de 12 niños tuvo un índice Kidmed medio de 6,83 , es decir, tuvieron una adherencia media a la Dieta 
Rev Esp Nutr Hum Diet. 2016; 20(1): 32 - 39

Valoración de la efectividad de la educación alimentaria en niños preescolares, padres y educadores

Tabla 2. Conocimientos sobre hábitos saludables de padres y profesores.

\begin{tabular}{|c|c|c|c|c|c|c|c|c|}
\hline \multirow[b]{3}{*}{ Pregunta } & \multicolumn{2}{|c|}{ Aciertos* } & \multicolumn{4}{|c|}{ Aciertos profesores* } & \multirow[b]{3}{*}{$\begin{array}{c}\mathrm{t} \\
\text { profesores }\end{array}$} & \multirow[b]{3}{*}{$\begin{array}{l}\text { Sig } \\
\text { profesores }\end{array}$} \\
\hline & Preintervención & Posintervención & & & Preintervención & Posintervención & & \\
\hline & media $\pm \mathrm{DT}$ & media $\pm D T$ & $\begin{array}{c}\mathrm{t} \\
\text { padres }\end{array}$ & $\begin{array}{c}\text { Sig } \\
\text { padres }\end{array}$ & media $\pm D T$ & media $\pm D T$ & & \\
\hline $\begin{array}{l}\text { 1. Lo más recomendable es } \\
\text { realizar } 5 \text { comidas al día }\end{array}$ & $0,67 \pm 0,49$ & $1 \pm 0,00$ & $-2,35$ & $<0,05$ & $0,63 \pm 0,52$ & $0,75 \pm 0,46$ & $-1,00$ & $>0,05$ \\
\hline $\begin{array}{l}\text { 2. Un desayuno saludable } \\
\text { está compuesto por un } \\
\text { lácteo, una fruta y un cereal }\end{array}$ & $0,50 \pm 0,52$ & $0,75 \pm 0,45$ & $-1,92$ & $>0,05$ & $0,63 \pm 0,52$ & $0,63 \pm 0,52$ & 0,00 & $>0,05$ \\
\hline $\begin{array}{l}\text { 3. Lo recomendable en una } \\
\text { alimentación saludable es } \\
\text { tomar } 3 \text { raciones de fruta } \\
\text { al día }\end{array}$ & $0,33 \pm 0,49$ & $0,92 \pm 0,29$ & $-3,92$ & $<0,01$ & $0,38 \pm 0,52$ & $0,75 \pm 0,46$ & $-1,43$ & $>0,05$ \\
\hline $\begin{array}{l}\text { 4. Lo recomendable en una } \\
\text { alimentación saludable es } \\
\text { tomar } 2 \text { raciones de verdura } \\
\text { al día }\end{array}$ & $0,67 \pm 0,49$ & $1 \pm 0,00$ & $-2,35$ & $<0,05$ & $1 \pm 0,00$ & $0,75 \pm 0,46$ & $-1,43$ & $>0,05$ \\
\hline $\begin{array}{l}\text { 5. Lo recomendable en una } \\
\text { alimentación saludable es } \\
\text { tomar pescado } 2 \text { veces a la } \\
\text { semana }\end{array}$ & $0,17 \pm 0,39$ & $1 \pm 0,00$ & $-7,42$ & $<0,001$ & $0,13 \pm 0,35$ & $0,50 \pm 0,54$ & $-2,05$ & $>0,05$ \\
\hline $\begin{array}{l}\text { 6. Lo recomendable en una } \\
\text { alimentación saludable } \\
\text { es tomar frutos secos al } \\
\text { menos } 3 \text { veces a la semana }\end{array}$ & $0,55 \pm 0,52$ & $0,91 \pm 0,30$ & $-1,79$ & $>0,05$ & $0,88 \pm 0,35$ & $0,63 \pm 0,52$ & 1,53 & $>0,05$ \\
\hline $\begin{array}{l}\text { 7. Lo recomendable en una } \\
\text { alimentación saludable es } \\
\text { tomar legumbres al menos } \\
2 \text { veces a la semana }\end{array}$ & $0,92 \pm 0,29$ & $0,75 \pm 0,45$ & 1,00 & $>0,05$ & $0,88 \pm 0,35$ & $1 \pm 0,00$ & $-1,00$ & $>0,05$ \\
\hline $\begin{array}{l}\text { 8. El aceite más saludable } \\
\text { es el de oliva }\end{array}$ & $1 \pm 0,00$ & $1 \pm 0,00$ & - & - & $1 \pm 0,00$ & $1 \pm 0,00$ & - & - \\
\hline $\begin{array}{l}\text { 9. Lo recomendable en una } \\
\text { alimentación saludable es } \\
\text { no tomar golosinas }\end{array}$ & $1 \pm 0,00$ & $0,92 \pm 0,29$ & 1,00 & $>0,05$ & $1 \pm 0,00$ & $1 \pm 0,00$ & - & - \\
\hline $\begin{array}{l}\text { 10. Es saludable realizar } \\
\text { alguna actividad física } \\
\text { diferente a caminar al } \\
\text { menos } 2 \text { veces a la semana }\end{array}$ & $0,92 \pm 0,29$ & $0,83 \pm 0,39$ & 0,56 & $>0,05$ & $1 \pm 0,00$ & $0,88 \pm 0,35$ & 1,00 & $>0,05$ \\
\hline Aciertos totales & $6,67 \pm 1,23$ & $8,83 \pm 0,84$ & $-6,29$ & $<0,001$ & $7,50 \pm 1,41$ & $7,75 \pm 1,17$ & $-0,68$ & $>0,05$ \\
\hline
\end{tabular}

$\mathbf{n}_{\text {padres }}=12 ; \mathbf{n}_{\text {profesores }}=8 ; *$ Codificación: $1=$ acierto, $0=$ fallo

Mediterránea, como refleja la Tabla 3. Tras la realización de una charla con los padres y un taller con los niños, el índice Kidmed de éstos ascendió a 8,50, pasando a tener una adherencia alta a la Dieta Mediterránea, aumento estadísticamente no significativo ( $t=-1,37 ; p>0,05)$. Al profundizar, se observó en los dos cuestionarios que todos los niños desayunaban, además incluían en esta comida un lácteo, en todas sus casas se utilizaba aceite de oliva y ninguno acudía una o más veces a un centro de comida rápida. Tras la intervención se consiguió disminuir el porcentaje de niños que tomaban golosinas y/o caramelos varias veces al día en un $9 \%$ $(t=-0,56 ; p>0,05)$. Por el contrario, se consiguió aumentar ligeramente el número de niños que tomaban verdura una vez al día en un $8 \%(t=-0,36 ; p>0,05)$ y más de una vez al día 
en un 9\% ( $t=-3,21 ; p>0,05)$; de mayor manera aumentó el número de niños que tomaban una primera y una segunda pieza de fruta al día en un $25 \%$ ( $t=-1,92 ; p>0,05)$ y $34 \%$ ( $t=-$ $1,77 ; p>0,05)$ respectivamente; y aumentó también en un $25 \%$ el número de niños que tomaban pasta o arroz casi a diario ( $t=-1,39 ; p>0,05)$, desayunaban un cereal o derivado $(t=-1,15 ; p>0,05)$, tomaban dos yogures $y / 040 \mathrm{~g}$ de queso al día ( $t=-1,39 ; p>0,05)$ y en un $34 \%$ los que tomaban frutos secos con regularidad ( $t=-1,77 ; p>0,05)$. Todos ellos aumentos no significativos $(p>0,05)$.

Respecto al número de comidas que tomaban los niños, antes de las intervenciones, el 83,3\% de la muestra realizaba cinco comidas al día, porcentaje que aumentó hasta el 100\% tras la intervención, aumento no significativo $(t=-1,48$; $p>0,05)$. En cuanto al tipo de desayuno, previo a la intervención ningún estudiante realizaba un desayuno completo, sin embargo, tras la intervención un 8,33\% lo realizaba, aumento que tampoco fue significativo $(t=-1,00 ; p>0,05)$.

Por último, al relacionar los conocimientos sobre hábitos saludables de los padres con la adherencia a la Dieta Mediterránea de sus hijos, se observó que los padres de los niños con mayor adherencia antes de la intervención mejoraron sus conocimientos sobre hábitos saludables después de la intervención educativa de manera significativa $(\rho=0,75$; $p<0,01)$.

Tabla 3. Test de Calidad de la Dieta Mediterránea en la Infancia y la Adolescencia.

\begin{tabular}{|c|c|c|c|c|}
\hline \multirow[b]{3}{*}{ Pregunta } & \multicolumn{2}{|c|}{ Aciertos } & \multirow[b]{3}{*}{$t$} & \multirow[b]{3}{*}{ Sig. } \\
\hline & Preintervención & Posintervención & & \\
\hline & media $\pm D T$ & media $\pm \mathrm{DT}$ & & \\
\hline Toma una fruta o un zumo natural todos los días & $0,67 \pm 0,49$ & $0,92 \pm 0,29$ & $-1,92$ & $>0,05$ \\
\hline Toma una $2^{a}$ pieza de fruta todos los días & $0,33 \pm 0,49$ & $0,67 \pm 0,49$ & $-1,77$ & $>0,05$ \\
\hline $\begin{array}{l}\text { Toma verduras frescas (ensaladas) o cocinadas regularmente } \\
\text { una vez al día }\end{array}$ & $0,50 \pm 0,52$ & $0,58 \pm 0,52$ & $-0,36$ & $>0,05$ \\
\hline $\begin{array}{l}\text { Toma verduras frescas o cocinadas de forma regular más de } \\
\text { una vez al día }\end{array}$ & $0,33 \pm 0,49$ & $0,42 \pm 0,52$ & $-3,21$ & $>0,05$ \\
\hline $\begin{array}{l}\text { Consume pescado con regularidad (por lo menos } 2-3 \text { veces a } \\
\text { la semana) }\end{array}$ & $0,92 \pm 0,29$ & $0,75 \pm 0,45$ & 1,00 & $>0,05$ \\
\hline $\begin{array}{l}\text { Acude una vez o más a la semana a un centro de comida } \\
\text { rápida (fast food) tipo hamburguesería }\end{array}$ & $0 \pm 0,00$ & $0 \pm 0,00$ & - & - \\
\hline $\begin{array}{l}\text { Le gustan las legumbres y las toma más de } 1 \text { vez a la } \\
\text { semana }\end{array}$ & $0,75 \pm 0,45$ & $0,75 \pm 0,45$ & 0,00 & $>0,05$ \\
\hline Toma pasta o arroz casi a diario (5 días o más a la semana) & $0,58 \pm 0,52$ & $0,83 \pm 0,39$ & $-1,39$ & $>0,05$ \\
\hline Desayuna un cereal o derivado (pan, etc.) & $0,50 \pm 0,52$ & $0,75 \pm 0,45$ & $-1,15$ & $>0,05$ \\
\hline $\begin{array}{l}\text { Toma frutos secos con regularidad (al menos } 2-3 \text { veces a la } \\
\text { semana) }\end{array}$ & $0,08 \pm 0,29$ & $0,42 \pm 0,52$ & $-1,77$ & $>0,05$ \\
\hline Se utiliza aceite de oliva en casa & $1 \pm 0,00$ & $1 \pm 0,00$ & - & - \\
\hline No desayuna & $0 \pm 0,00$ & $0 \pm 0,00$ & - & - \\
\hline Desayuna un lácteo (yogurt, leche, etc.) & $1 \pm 0,00$ & $1 \pm 0,00$ & - & - \\
\hline Desayuna bollería industrial, galletas o pastelitos & $0,33 \pm 0,49$ & $0,33 \pm 0,49$ & 0,00 & $>0,05$ \\
\hline Toma 2 yogures y/o 40 g de queso cada día & $0,58 \pm 0,52$ & $0,83 \pm 0,39$ & $-1,39$ & $>0,05$ \\
\hline Toma golosinas y/o caramelos varias veces al día & $0,17 \pm 0,39$ & $0,08 \pm 0,29$ & $-0,56$ & $>0,05$ \\
\hline Puntuación Kidmed total & $6,83 \pm 2,29$ & $8,50 \pm 2,84$ & $-1,37$ & $>0,05$ \\
\hline
\end{tabular}




\section{$\longrightarrow$ \\ DISCUSIÓN}

Según reflejan estudios anteriores, con intervenciones llevadas a cabo por profesionales sanitarios en niños de mayor edad, se consigue un aumento significativo en determinados conocimientos nutricionales y hábitos de vida saludables $^{12,13}$. En este estudio, el grupo de padres de los niños inicialmente con mayor adherencia a la Dieta Mediterránea, aumenta de manera significativa sus conocimientos sobre hábitos saludables tras las intervenciones, quizá más motivados por temas relacionados con la salud o la nutrición, elemento fundamental en el aprendizaje ${ }^{18}$.

En cuanto al grado de adherencia a la Dieta Mediterránea, anteriormente, el estudio enKid mostró que la mayoría de la población española entre 2 y 14 años, un 48,6\%, tenía una adherencia media ${ }^{19}$. En concreto en la zona de Levante, la mitad de la población, un 50,2\%, entre 2 y 24 años mostraba este grado de adherencia ${ }^{19}$; datos similares a los encontrados en este estudio inicialmente, donde un 58,33\% de la muestra también tiene un índice Kidmed entre 4 y 7. Además, en estudios realizados con niños de mayor edad se observa que al aumentar la edad disminuye la adherencia $^{11,20-23}$. Aunque de manera no significativa, en este estudio se ha producido tras las intervenciones un aumento en la adherencia a la Dieta Mediterránea. En estudios posteriores sería necesario comprobar que este aumento se mantiene en el tiempo, ya que los hábitos alimentarios están influenciados por múltiples factores ${ }^{24}$. En cuanto a la distribución de tomas, el estudio enKid mostró que aproximadamente el $40 \%$ de su muestra de niños entre 2-5 años realizaba cinco comidas diarias ${ }^{25}$; datos alejados a los encontrados en el presente estudio, donde tras las intervenciones todos los niños realizan cinco comidas al día. Teniendo así más posibilidades de conseguir un adecuado reparto energético. No obstante, las recomendaciones de un desayuno completo, o como refleja el estudio enKid, desayuno de muy buena calidad, integrado por un lácteo, un cereal y una fruta ${ }^{26}$, en un estudio anterior realizado con niños de 3-16 años es seguido por un $33 \%$ de la muestra ${ }^{27}$. Porcentaje muy alejado del encontrado en el presente estudio, donde tras las intervenciones fue realizado por el $8,33 \%$ de los niños. Lo que manifiesta que se trata de un hábito débilmente instaurado que necesita una mayor dedicación para modificarlo.

El presente estudio tiene como limitación la evaluación de la intervención a corto plazo, en futuras intervenciones debería realizarse una evaluación de los hábitos saludables a largo plazo. Otra limitación puede ser la participación de padres ya sensibilizados con este tema, por lo que los resultados de los hábitos alimentarios iniciales pueden ser superiores a la media de la población general.

En próximos estudios sería interesante evaluar si los niños cuyos padres participan en la intervención mejoran sus conocimientos sobre hábitos saludables respecto a los niños cuyos padres no colaboran, para conocer la influencia que ejercen los padres respecto a estos conocimientos, en sus hijos.

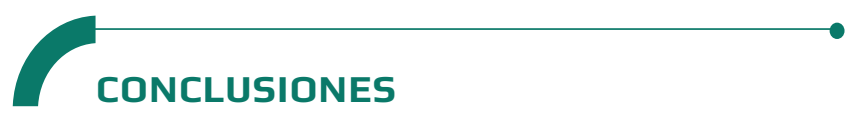

Sólo se observa un aumento significativo de conocimientos en los niños para el caso de la actividad física. En futuras intervenciones deberían llevarse a cabo actividades promotoras de hábitos saludables que incluyan la alimentación y actividad física por Dietistas-Nutricionistas a lo largo de todo el curso académico en niños de edades tan tempranas, contando con la colaboración de sus profesores y adecuando el material educativo a su nivel de comprensión. En cuanto a los padres, sí se ha conseguido un aumento significativo en la adquisición general de conocimientos así como en el número de comidas y frecuencia de consumo de fruta, verdura y pescado tras las dos intervenciones educativas. Partir de unos conocimientos adecuados es necesario para transmitir unos hábitos alimentarios saludables y poder aplicarlos en la alimentación diaria. Para ello es necesaria la realización de programas de educación alimentaria por parte de Dietistas-Nutricionistas dirigidos a los responsables de la alimentación infantil, para mejorar su alimentación, como método de promoción de la salud.

\section{CONFLICTO DE INTERESES}

Los autores expresan que no hay conflictos de intereses al redactar el manuscrito.

\section{BIBLIOGRAFÍA}

(1) Organización Mundial de la Salud. Dieta, nutrición y prevención de enfermedades crónicas: informe de una Consulta Mixta de Expertos OMS/FAO. Ginebra, Suiza: OMS; 2003. Report No.: 916.

(2) Serra L, Ribas L. Hábitos alimentarios y consumo de alimentos en España: Dieta Mediterránea. En: Nutrición y salud pública: métodos, bases científicas y aplicaciones. 1a. ed. Barcelona: Masson; 1995. p. 303-10.

(3) Aranceta J. Nutrición en la edad evolutiva. En: Nutrición y salud pública: métodos, bases científicas y aplicaciones. 1a. ed. 
Barcelona: Masson; 1995. p. 185-92.

(4) Macias Al, Gordillo LG, Camacho EJ. Hábitos alimentarios de niños en edad escolar y el papel de la educación para la salud. Rev Chil Nutr. 2012; 39(3): 40-3.

(5) Fundación Dieta Mediterránea. ¿Qué es la Dieta Mediterránea? [Internet]. Fundación Dieta Mediterránea. [citado 2 de julio de 2015]. Recuperado a partir de: http://dietamediterranea. com/nutricion-saludable-ejercicio-fisico/

(6) Castro-Quezada I, Román-Viñas B, Serra-Majem L. The Mediterranean diet and nutritional adequacy: a review. Nutrients. 2014; 6(1): 231-48.

(7) Estruch R, Ros E, Salas-Salvadó J, Covas M-I, Corella D, Arós $F$, et al. Primary prevention of cardiovascular disease with a Mediterranean diet. N Engl J Med. 2013; 368(14): 1279-90.

(8) Durá Travé T, Castroviejo Gandarias A. Adherencia a la dieta mediterránea en la población universitaria. Nutr Hosp. 2011; 26(3): 602-8.

(9) Martínez-González MA, García-López M, Bes-Rastrollo M, Toledo E, Martínez-Lapiscina EH, Delgado-Rodriguez M, et al. Mediterranean diet and the incidence of cardiovascular disease: a Spanish cohort. Nutr Metab Cardiovasc Dis. 2011; 21(4): 237-44.

(10) Pérez Gallardo L, Bayona I, Mingo T, Rubiales C. Utilidad de los programas de educación nutricional para prevenir la obesidad infantil a través de un estudio piloto en Soria. Nutr Hosp. 2011; 26(5): 1161-7.

(11) Mariscal-Arcas M, Rivas A, Velasco J, Ortega M, Caballero AM, Olea-Serrano F. Evaluation of the Mediterranean Diet Quality Index (KIDMED) in children and adolescents in Southern Spain. Public Health Nutr. 2009; 12(9): 1408-12.

(12) Oliva Rodríguez R, Tous Romero M, Gil Barcenilla B, Longo Abril G, Pereira Cunill JL, García Luna PP. Impacto de una intervención educativa breve a escolares sobre nutrición y hábitos saludables impartida por un profesional sanitario. Nutr Hosp. 2013; 28(5): 1567-73.

(13) Calleja Fernández A, Muñoz Weigand C, Ballesteros Pomar MD, Vidal Casariego A, López Gómez J], Cano Rodríguez I, et al. Modificación de los hábitos alimentarios del almuerzo en una población escolar. Nutr Hosp. 2011; 26(3): 560-5.

(14) Jefatura del Estado. Ley 41/2002, de 14 de noviembre, básica reguladora de la autonomía del paciente y de derechos y obligaciones en materia de información y documentación clínica. BOE núm. 274. Sec. I. Disposiciones generales 2002 p. 40126-32.

(15) Sociedad Española de Nutrición Comunitaria, Sociedad Española de Medicina de Familia y Comunitaria, editores. 1. Planificación de una alimentación saludable. En: Consejos para una Alimentación Saludable. Madrid, España: SENC; 2007. p. 10-4.

(16) Alonso C, López P, de la Cruz O. Creer tocando. Tendencias Pedagógicas. 2013; 21: 249-62.

(17) Serra-Majem L, Ribas L, Ngo J, Ortega RM, García A, Pérez-
Rodrigo C, et al. Food, youth and the Mediterranean diet in Spain. Development of KIDMED, Mediterranean Diet Quality Index in children and adolescents. Public Health Nutr. 2004; 7(7): 931-5.

(18) Ospina Rodríguez J. La motivación, motor del aprendizaje. Rev Cienc Salud. 2006; 4(2): 158-60.

(19) Serra L, Ribas L, Ngo J, Ortega RM, Pérez-Rodrigo C, Aranceta J. 5. Alimentación, jóvenes y dieta mediterránea en España. Desarrollo del KIDMED, índice de calidad de la dieta mediterránea en la infancia y la adolescencia. En: Alimentación infantil y juvenil. Barcelona: Masson; 2002.

(20) Ayechu Díaz A, Durá Travé T. Dieta mediterránea y adolescentes. Nutr Hosp. 2009; 24(6): 759-60.

(21) Ayechu A, Durá T. Calidad de los hábitos alimentarios (adherencia a la dieta mediterránea) en los alumnos de educación secundaria obligatoria. An Sist Sanit Navar. 2010; 33(1): 35-42.

(22) Grao-Cruces A, Nuviala A, Fernández-Martínez A, Porcel-Gálvez A-M, Moral-García J-E, Martínez-López E-J. Adherencia a la dieta mediterránea en adolescentes rurales y urbanos del sur de España, satisfacción con la vida, antropometría y actividades físicas y sedentarias. Nutr Hosp. 2013; 28(4): 1129-35.

(23) Navarro-Solera M, González-Carrascosa R, Soriano JM. Estudio del estado nutricional de estudiantes de educación primaria y secundaria de la provincia de Valencia y su relación con la adherencia a la Dieta Mediterránea. Rev Esp Nutr Hum Diet. 2014; 18(2): 81-8.

(24) Dárdano C, Álvarez C. El Ambiente Alimentario en las Escuelas, las Políticas de Alimentación Escolar y la Educación en Nutrición [Internet]. Roma, Italia: Grupo de Educación Nutricional y Protección del Consumidor de la FAO; 2011 [citado 2 de julio de 2015]. Recuperado a partir de: http:// www.fao.org/ag/humannutrition/29281-099a2c34289e1039 5c79079637288c843.pdf

(25) Serra L, Ribas L, García R, Pérez-Rodrigo C, Peña L, Aranceta J. 2. Hábitos alimentarios y consumo de alimentos en la población infantil y juvenil española (1998-2000): variables socioeconómicas y geográficas. En: Alimentación infantil y juvenil. Barcelona: Masson; 2002.

(26) Serra Majem L, Ribas Barba L, Aranceta Bartrina J, Pérez Rodrigo C, Saavedra Santana P, Peña Quintana L. Obesidad infantil y juvenil en España. Resultados del Estudio enKid (1998-2000). Med Clin. 2003; 121(19): 725-32.

(27) Aranceta Bartrina J, Pérez Rodrigo C, Serra Majem L, Delgado Rubio A. Hábitos alimentarios de los alumnos usuarios de comedores escolares en España. Estudio «Dime Cómo Comes». Aten Primaria. 2004; 33(3): 131-9. 